Supplement of Atmos. Chem. Phys. Discuss., 15, 24839-24870, 2015

http://www.atmos-chem-phys-discuss.net/15/24839/2015/

doi:10.5194/acpd-15-24839-2015-supplement

(C) Author(s) 2015. CC Attribution 3.0 License.

(c) (i)

\title{
Top-down estimates of benzene and toluene emissions in Pearl River Delta and Hong Kong, China
}

\section{Fang et al.}

Correspondence to: X. Fang (fangxk@mit.edu)

The copyright of individual parts of the supplement might differ from the CC-BY 3.0 licence. 
21 Table S1. Performance comparison of simulating benzene and toluene mixing ratios at the 22 Heshan site using the gridded emissions estimated by RCP 3PD, Yin et al. 2015, REAS v1.1

23 REF, MEIC v1.2 and this study. $\boldsymbol{E}$ is the root mean square error (RMSE) for the bias between the 24 simulated and observed mixing ratios. $\boldsymbol{B}$ denotes the mean bias between the simulated and 25 observed mixing ratios. $\boldsymbol{r}^{2}$ represents the squared Pearson correlation coefficients between the 26 simulated and observed mixing ratios.

\begin{tabular}{ccccccc}
\hline & \multicolumn{3}{c}{ Benzene simulation } & \multicolumn{3}{c}{ Toluene simulation } \\
\cline { 2 - 7 } & $E$ & $B$ & $r^{2}$ & $E$ & $B$ & $r^{2}$ \\
\hline RCP 3PD & 1.396 & 0.783 & 0.195 & 5.80 & 4.65 & 0.054 \\
Yin et al. 2015 & 1.489 & 0.645 & 0.244 & 5.30 & 4.02 & 0.069 \\
REAS v1.1 & 2.237 & 1.871 & 0.083 & 5.67 & 4.44 & 0.023 \\
MEIC v1.2 & 1.491 & 0.941 & 0.198 & 5.00 & 1.56 & 0.042 \\
This study & 1.262 & 0.409 & 0.268 & 4.30 & 1.99 & 0.097 \\
This study performed best? & Yes & Yes & Yes & Yes & No & Yes \\
\hline
\end{tabular}



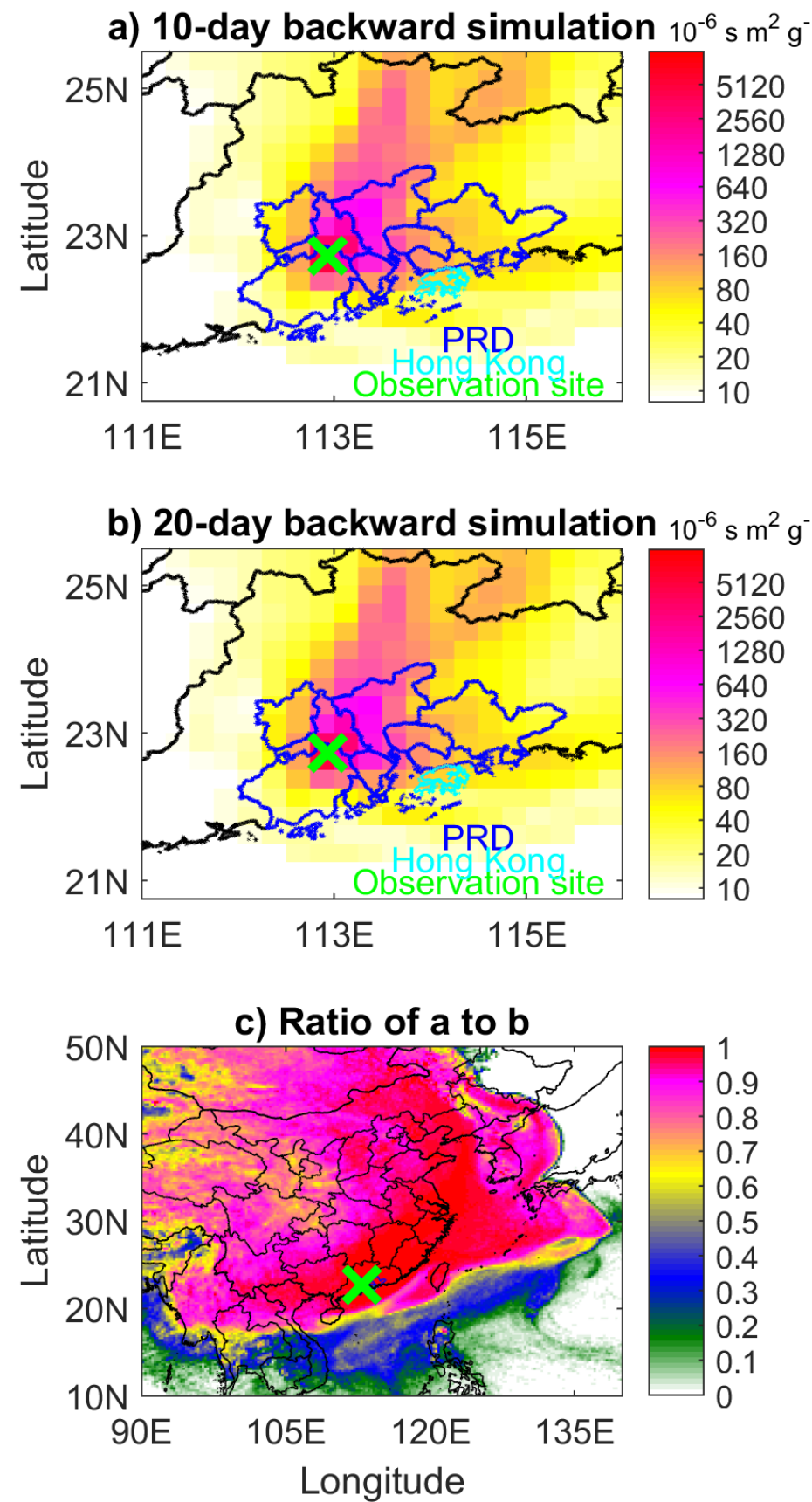

28 Figure S1. Spatial distribution of the average benzene emission sensitivities a) from 10-day backward 29 simulation, b) from 20-day backward simulation, and c) the ratio of a to b for the observation period. Note 30 that the domain size in c) is not the same as in a) and b). 

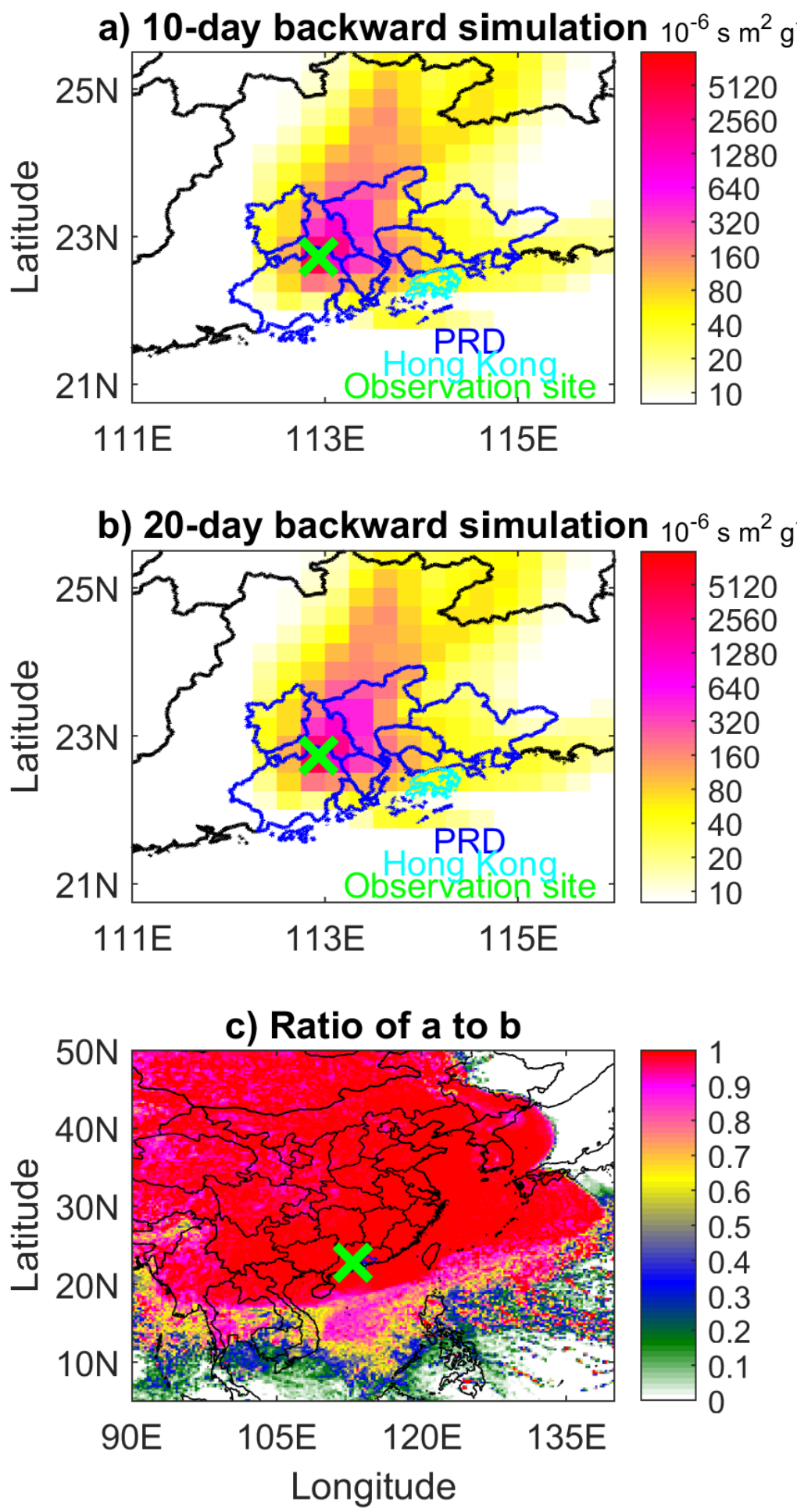

Figure S2. Spatial distribution of the average toluene emission sensitivities a) from 10-day backward

33 simulation, b) from 20-day backward simulation, and c) the ratio of a to $\mathrm{b}$ for the observation period. Note that the domain size in c) is not the same as in a) and b). 

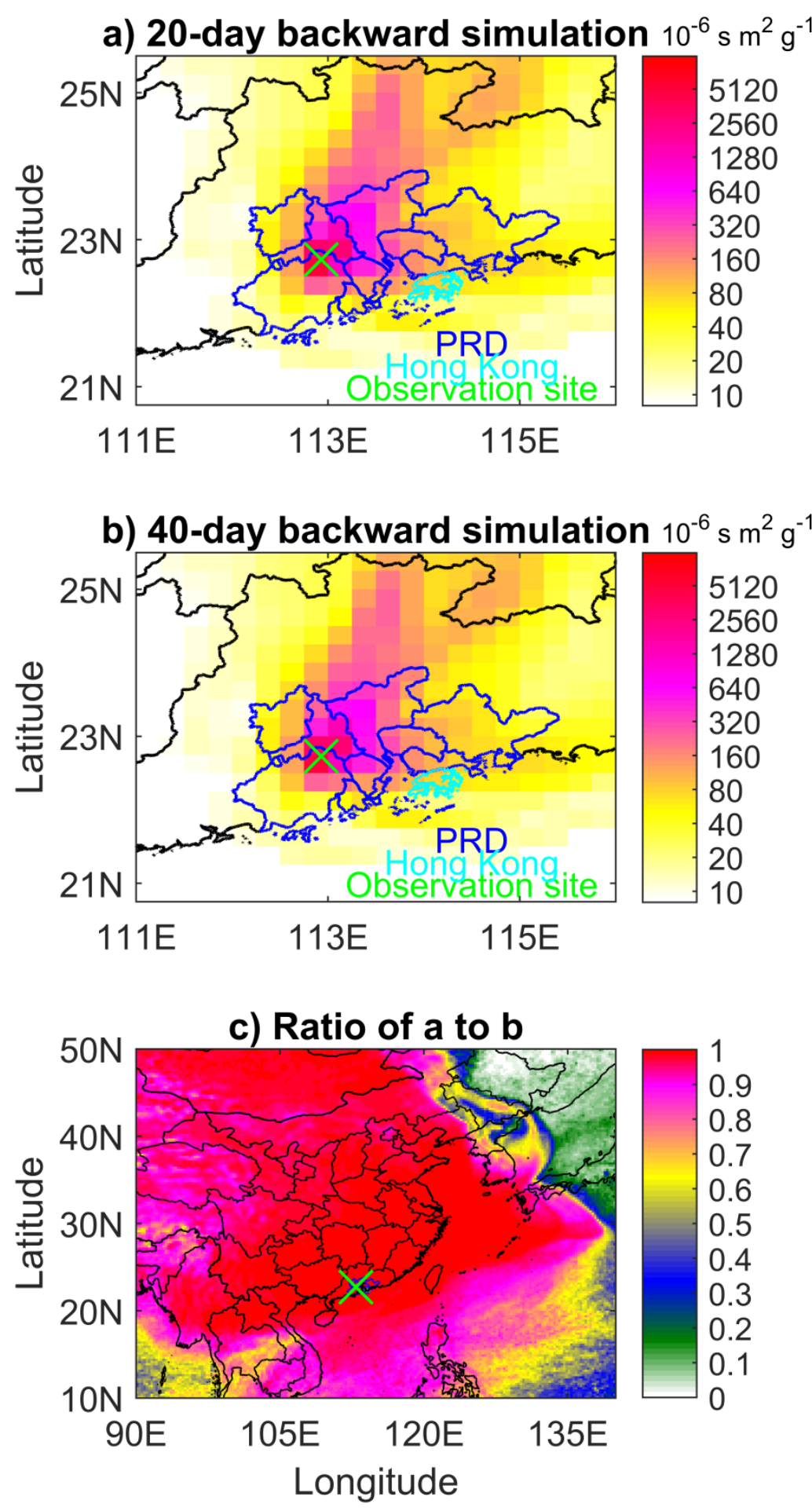

36 Figure S3. Spatial distribution of the average benzene emission sensitivities a) from 20-day backward

37 simulation, b) from 40-day backward simulation, and c) the ratio of a to $b$ for the observation period. Note 38 that the domain size in c) is not the same as in a) and b). 

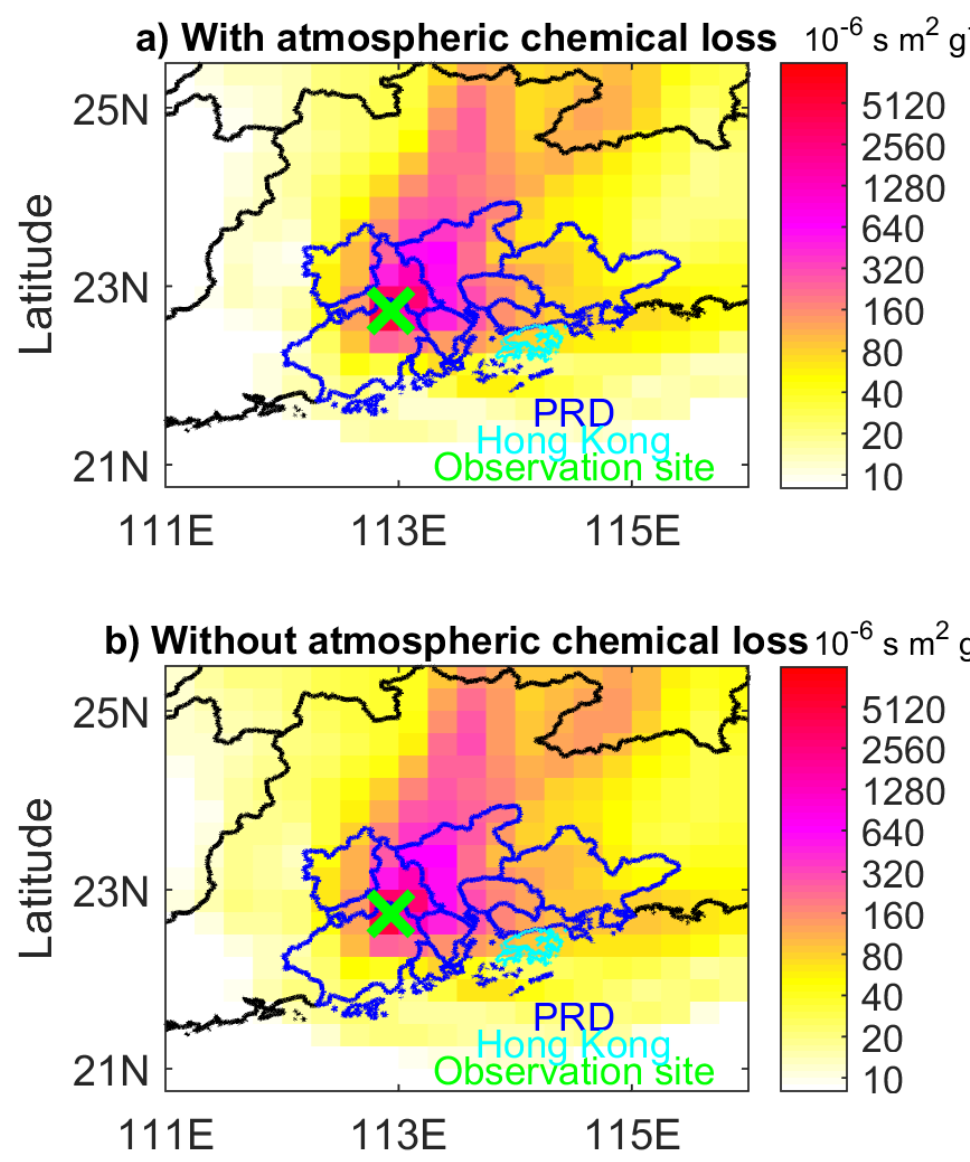

c) Ratio of $a$ to $b$

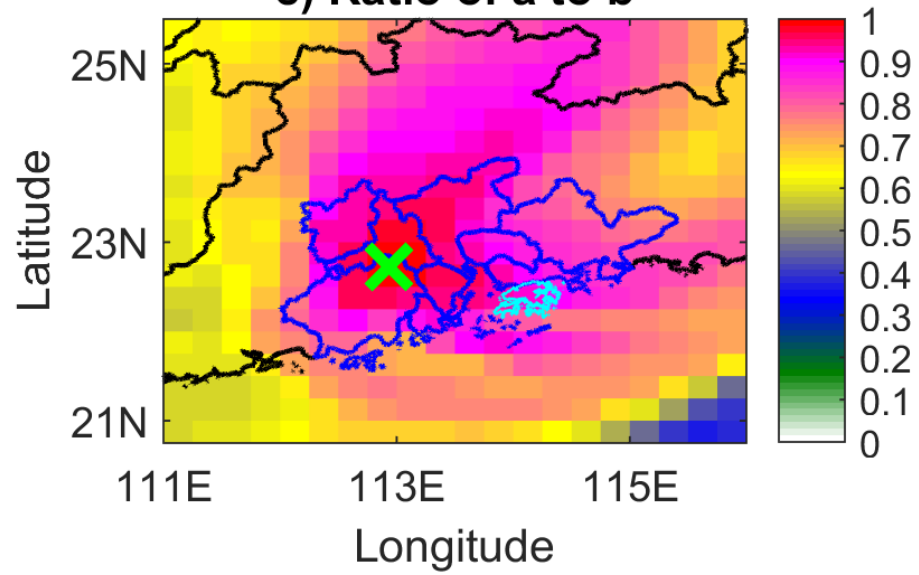

40 Figure S4. Spatial distribution of the average toluene emission sensitivities from a) with atmospheric 41 chemical loss, b) without atmospheric chemical loss, and c) the ratio of a to $b$ for the observation period. 

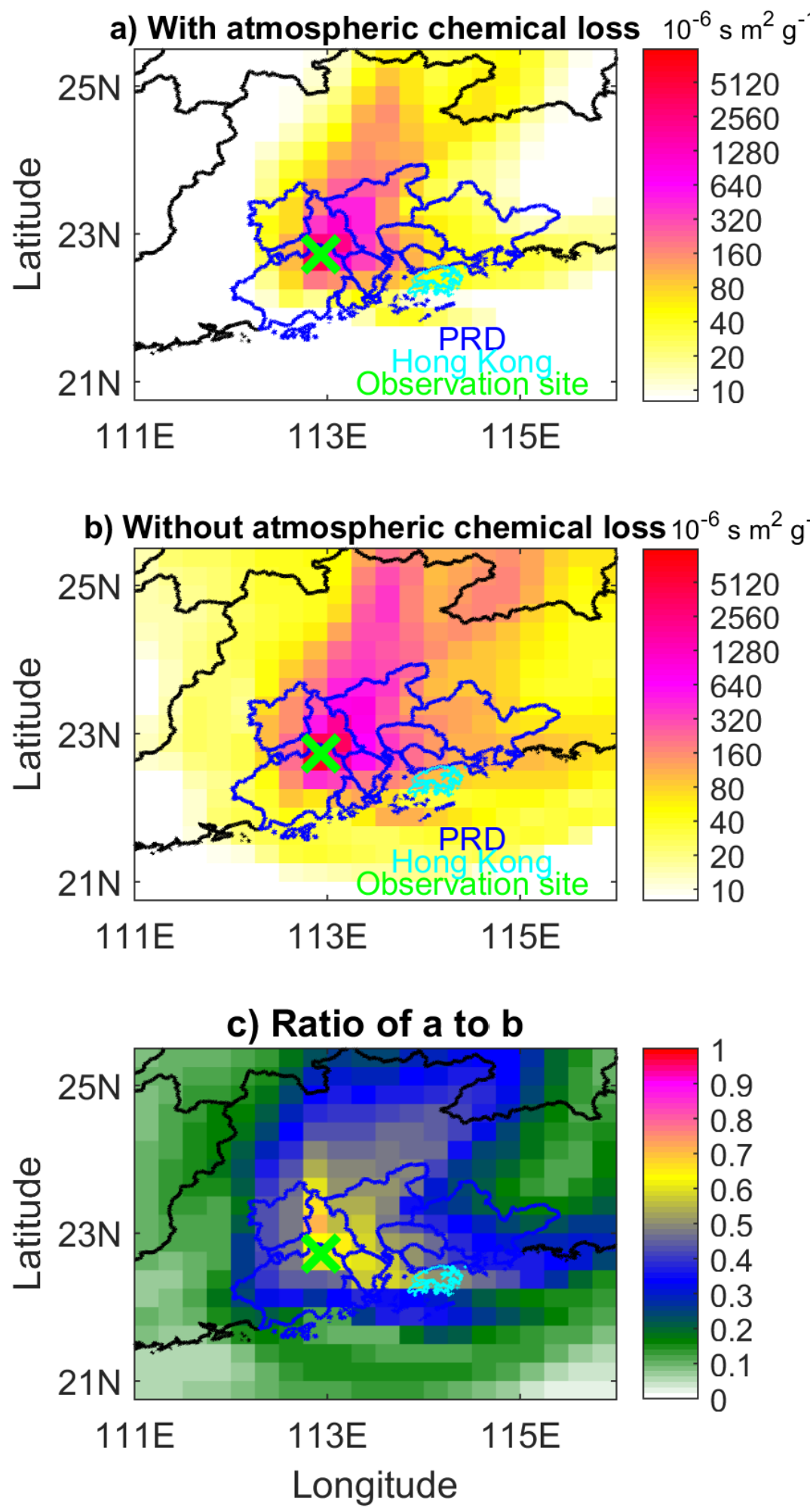

43 Figure S5. Spatial distribution of the average toluene emission sensitivities from a) with atmospheric 44 chemical loss, b) without atmospheric chemical loss, and c) the ratio of a to $b$ for the observation period. 


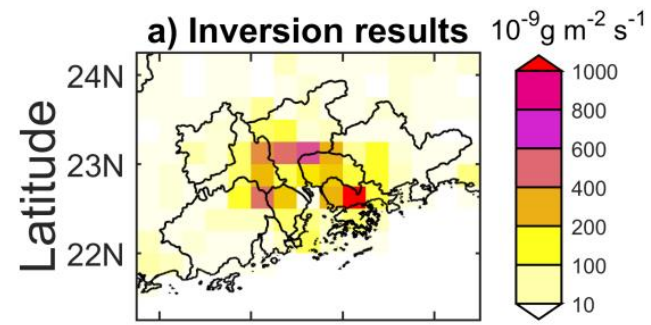

\section{Toluene}
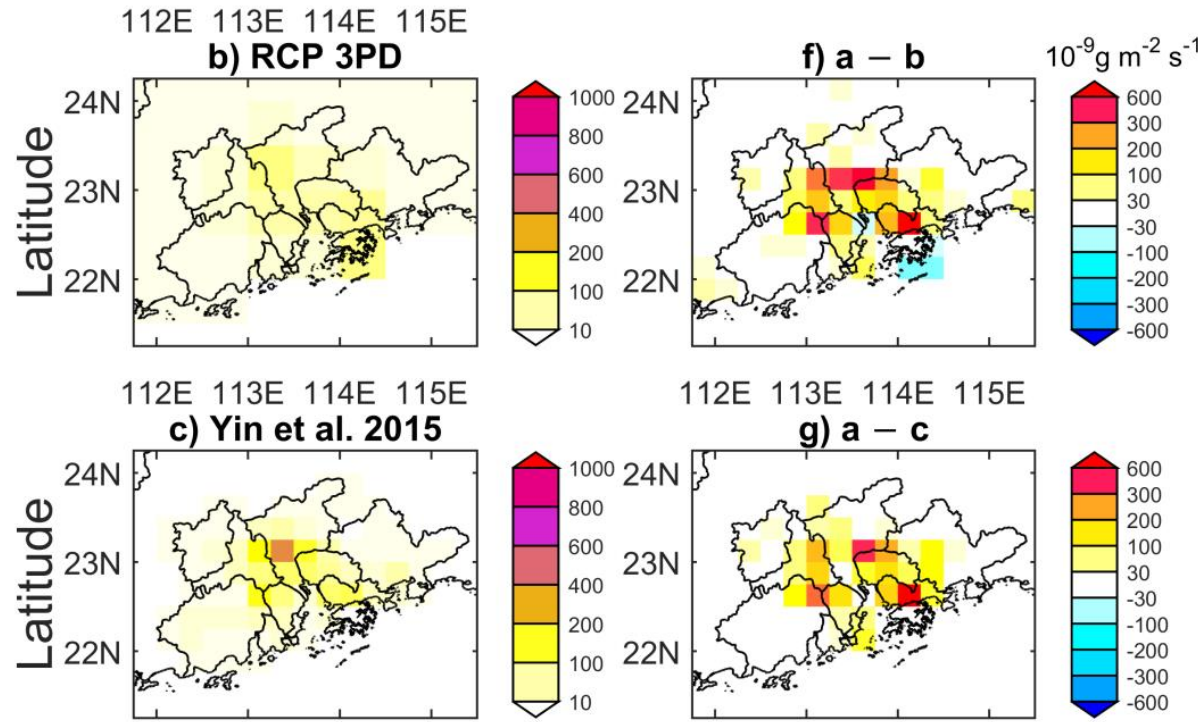

112E 113E 114E 115E

112E 113E 114E 115E
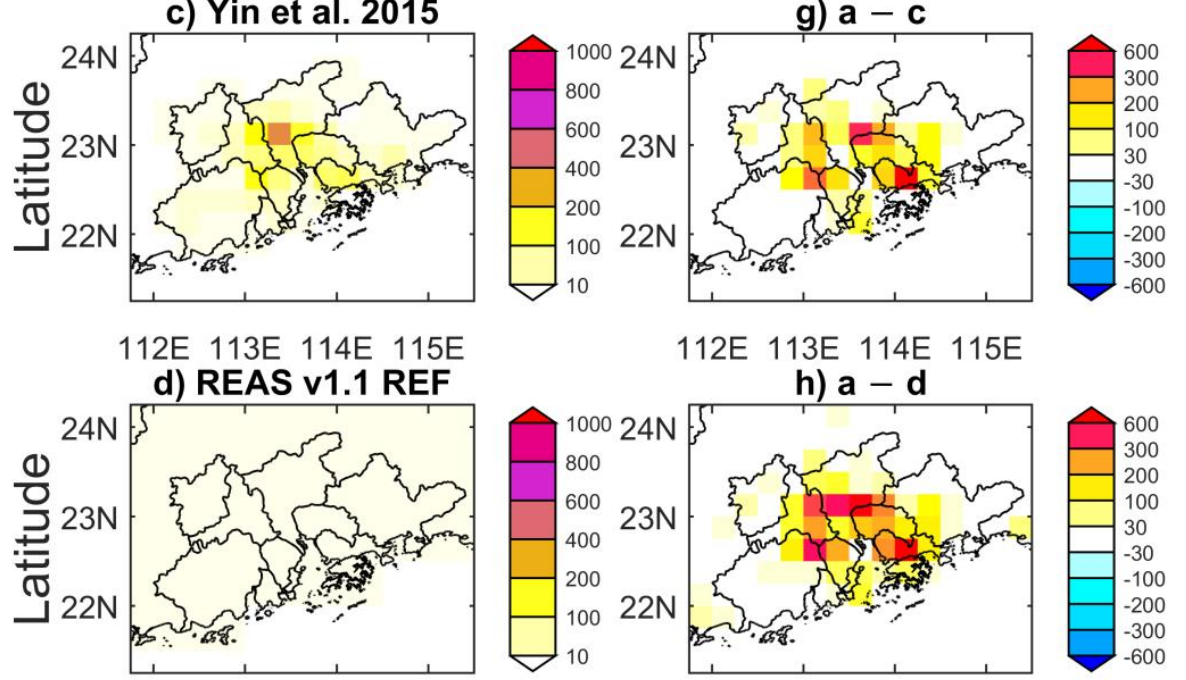

112E 113E 114E 115E

h) $\mathbf{a}-\mathbf{d}$

112E 113E 114E 115E e) MEIC v1.2

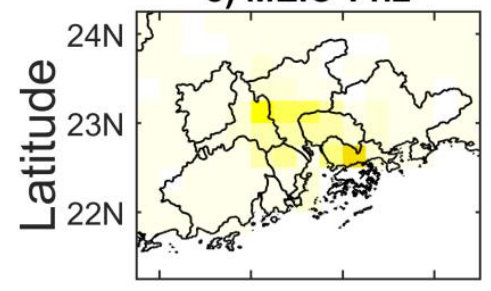

112E 113E 114E 115E
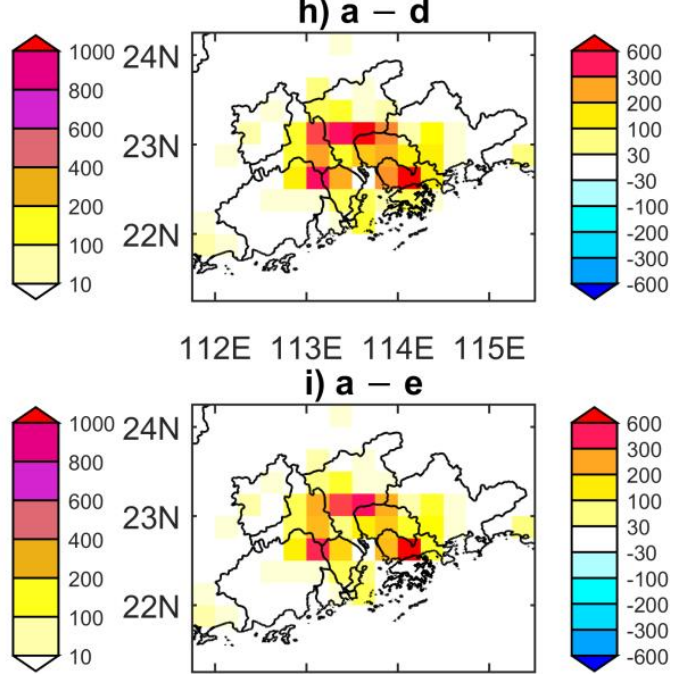

112E 113E 114E 115E i) $\mathbf{a}-\mathbf{e}$

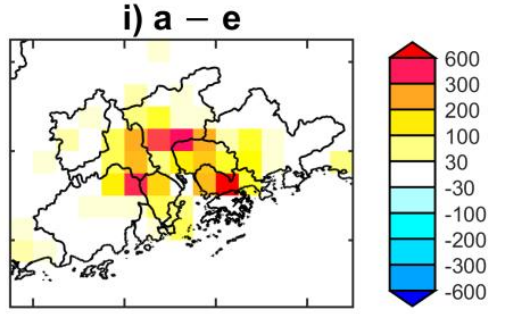

112E 113E 114E 115E

\section{Longitude}

\section{Longitude}

Figure S6. Maps of toluene emissions for PRD, HK and surrounding regions from a) inversion, b) RCP 3PD, c) Yin et al. (2015), d) REAS v1.1 REF, e) MEIC v1.2, and the difference between inversion results (a) and the bottom-up inventories (b, c, d, e). Note that in c) and g) only emissions within PRD are plotted since Yin et al. (2015) only estimated emissions within PRD, and that in e) and i) emissions within HK are not plotted since MEIC v1.2 has not estimated toluene emission in HK. 


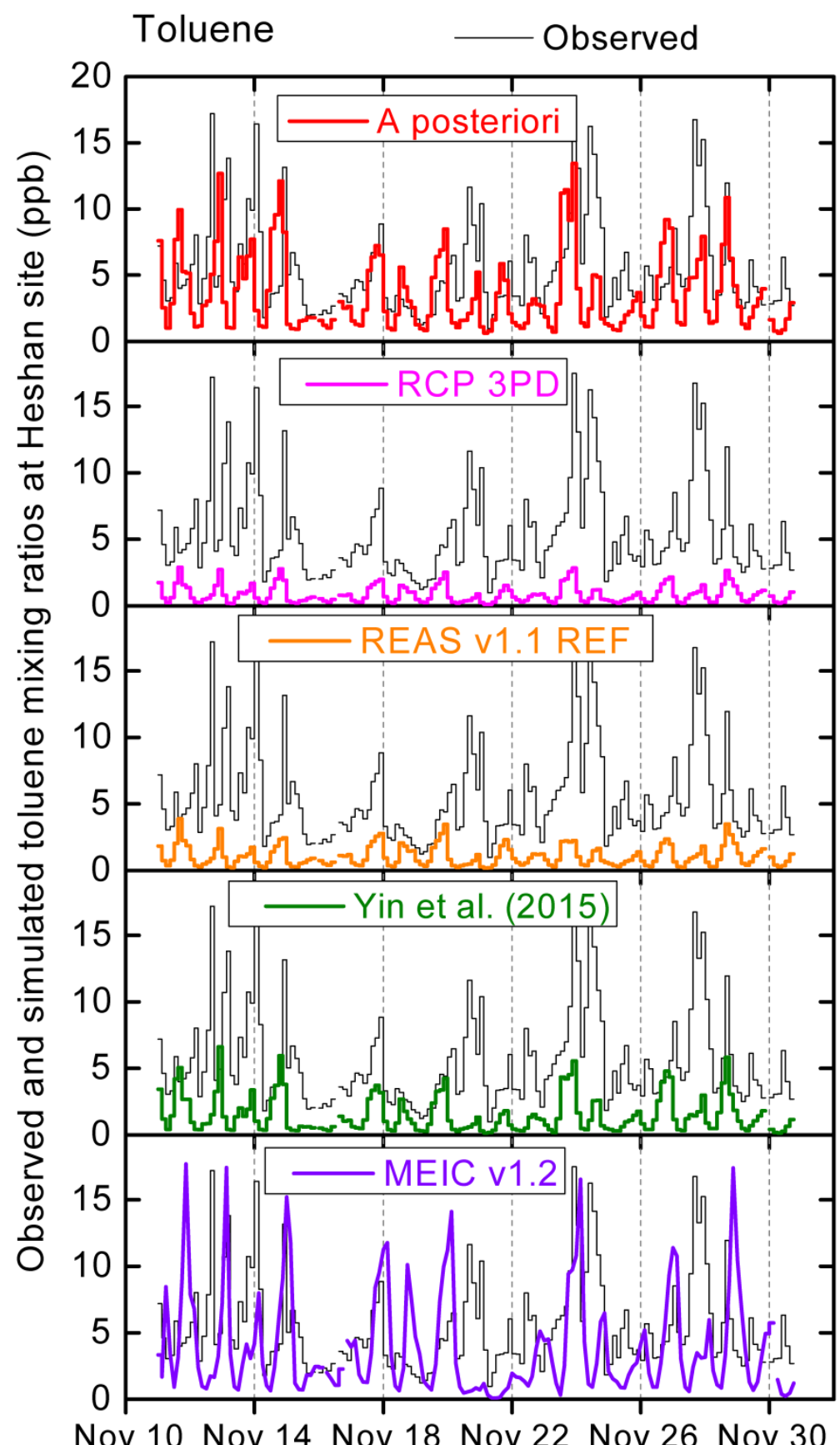

53 Figure S7. Time series of observed and simulated toluene mixing ratios at the Heshan site. The

54 simulations use emission fields from inversion in this study, RCP 3PD, REAS v1.1 REF, Yin et al. (2015)

55 and MEIC v1.2, respectively. 